\title{
Familial aggregation of anxiety associated with bruxism
}

David Gorski $^{1}$

${ }^{1}$ University of Pittsburgh School of Dental Medicine, Pittsburgh PA, USA

\section{Abstract}

Background: This paper reports the co-occurrence of heritable anxiety-related disorders and awake bruxism in a family and discusses the heritability of anxiety and implications of awake bruxism as comorbidity to anxiety disorders.

Subjects: Ten out of 14 members of an extended family reported having a professionally diagnosed anxiety-related disorder. All individuals with anxiety disorders also showed intraoral signs of wear from bruxism and reported being aware of grinding their teeth while awake. Additionally, three out of the four family members without an anxiety-related disorder did not report grinding their teeth and showed no occlusal wear from bruxism. All of the individuals that were examined were educated about the short-term and long-term complications associated with bruxism, but all of them elected to not have treatment performed.

Practical Implications: Anxiety disorders are highly related to suicidal behaviors, particularly in children and adolescents. Additionally, awake bruxism can often serve as an indicator of anxiety or stress. By recognizing bruxism as a possible manifestation of psychological distress, the dental practitioner may be able to direct patients to life-saving services like psychologists and crisis hotlines when appropriate.
Citation: Gorski D. (2015) Familial aggregation of anxiety associated with bruxism. Dentistry 3000. 1:a001 doi:10.5195/d3000.2015.34

Received: June 2, 2015

Accepted: June 26, 2015

Published: July 20, 2015

Copyright: (C2015 Gorski D. This is an open access article licensed under a Creative Commons Attribution Work 4.0 United States License. Email: deg32@pitt.edu

\section{Introduction}

Bruxism is defined as "a repetitive jaw-muscle activity characterized by clenching or grinding of the teeth and/or by bracing or thrusting of the mandible" that can occur while asleep (sleep bruxism) or while awake (awake bruxism) [1]. Awake bruxism is reported in $20 \%$ of the adult population in the US [2], but researchers have only recently linked its occurrence to anxiety disorders [3].

The heritability estimates for anxiety disorders range from $20 \%$ to $65 \%$ [4]. From family and twin studies, the heritability of Generalized Anxiety Disorder (GAD) has been measured at $32 \%$, indicating significant heritability for this anxiety-based condition [5].

Awake bruxism and anxiety are often only considered in their respective fields of dentistry and psychology. This report describes the cooccurrence of anxiety disorders and their manifestation as awake bruxism in family, and it discusses the clinical implications of these findings.

\section{Subjects}

A 28 year-old male was complaining of "jaw pain" and "headaches" that had been worsening in the past two years. The patient had a relatively unremarkable medical history. Other than minor seasonal allergies for which he was taking no medications, his only notable conditions were Generalized Anxiety Disorder and Major Depressive Disorder. For these conditions, he was

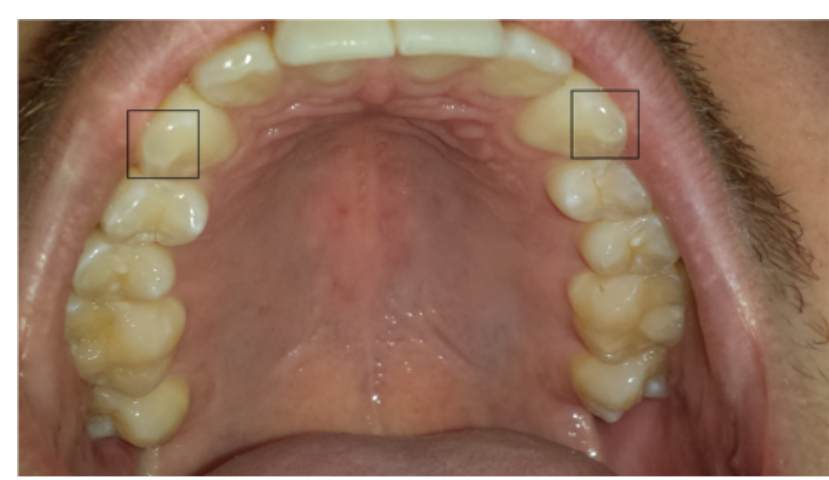

Figure 1. Proband's maxillary dentition. Note the wear facets on the maxillary canines. 


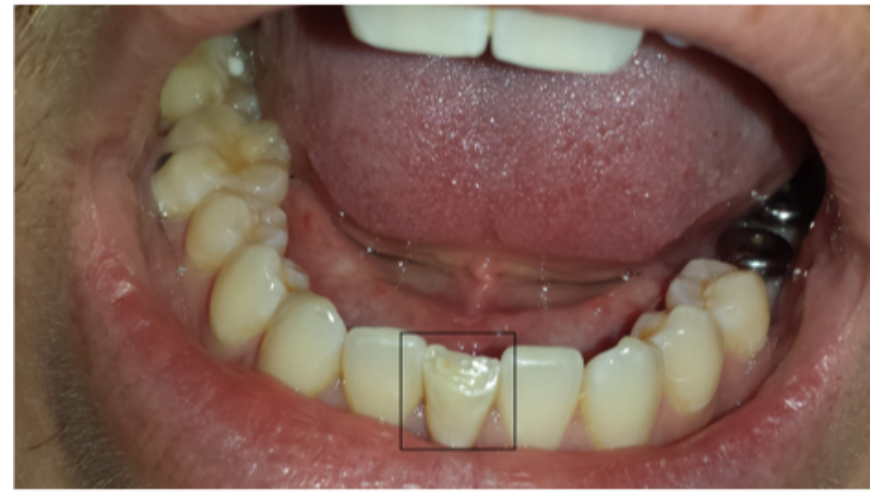

Figure 2. Proband's mandibular dentition. Note the occlusal wear from bruxism on the mandibular central incisor. had provided some relief from the headaches and jaw pain.

Figures 3 and 4 show the phenotypical distribution in the family after oral examinations and medical history interviewing of the proband's family. taking Pristiq, a selective serotonin and norepinephrine reuptake inhibitor, and Lithium Carbonate, a pharmaceutical typically used to

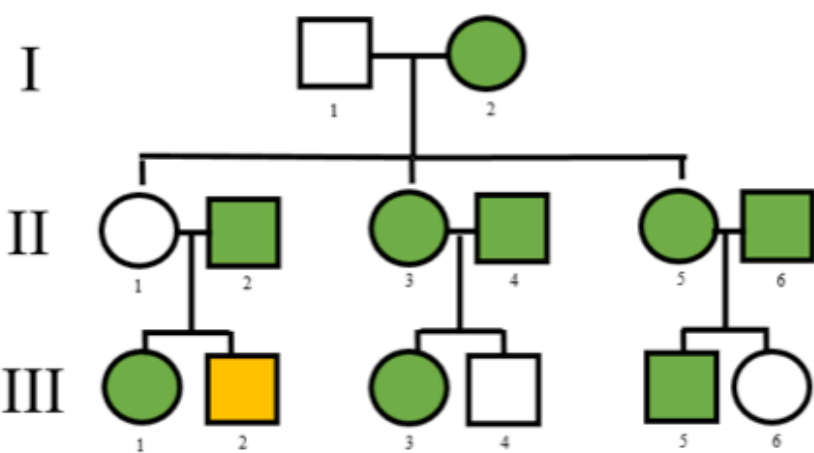

Figure 3. Pedigree of the family indicating the affected by anxiety-related disorders. Proband is marked in yellow. The rest of affected individuals are marked in green.

treat bipolar disorder. A thorough head and neck exam with inspection of hard and soft tissues showed bilateral sensitivity of the masseter and medial pterygoid muscles, with well-defined wear facets on the incisal and lingual surfaces of his maxillary canines (Figure 1). Additionally, trauma from occlusal wear was noted on his single mandibular incisor (Figure 2). When questioned, the patient stated that he was aware that he had been grinding his teeth during the day. He recalled that his sister had recently been having similar symptoms and had received a "night guard" that

awkward" to wear but did not wish
Out of 13 individuals examined, ten had professional diagnoses of anxiety-related conditions and also showed intraoral signs of bruxism (Figures 5, 6, and 7). Additionally, out of the four family members who did not have an anxiety-related condition, only individual II.1 showed any intraoral signs of bruxism or admitted to "grinding or clenching their

teeth" (Figure 8).

None of the family members that were inspected wished to have treatment performed. Individuals III.1 and II.4 already had occlusal splints that they wore at night. They both rated their symptom relief from their occlusal splints as "moderate." Individual II.4 said that his occlusal splint was "uncomfortable and

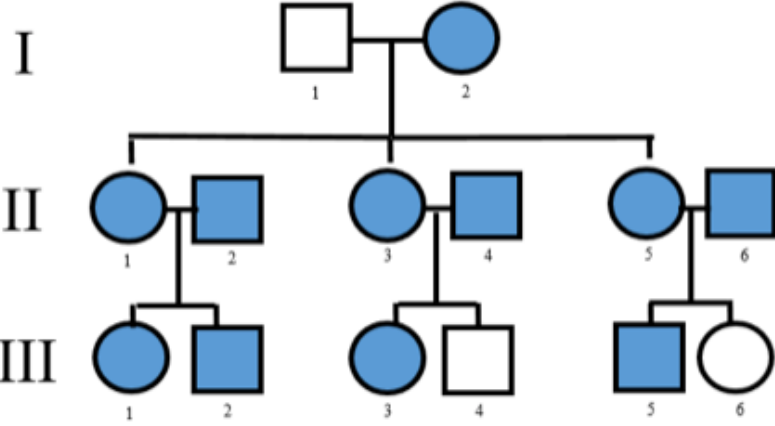

Figure 4. Pedigree of the family indicating the affected by bruxism. Note that all individuals that have an anxietyrelated disorder also brux. 
Awake bruxism has been noted to have a number of varied associations-smoking, caffeine intake, and alcohol consumption [8]. However, in a study of 86 children by Monaco

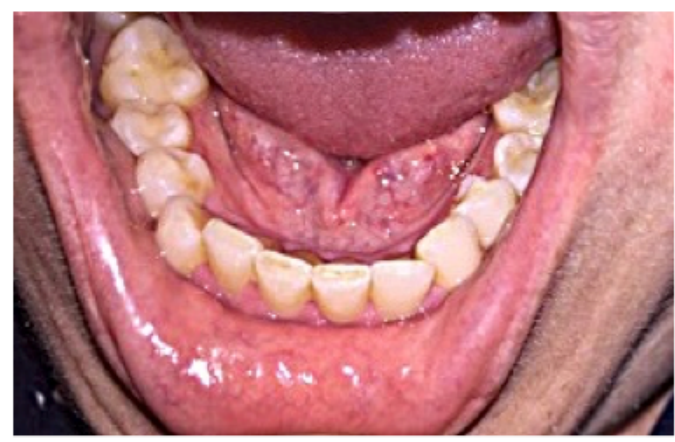

Figure 5. Intraoral signs of bruxism in individual III.1. et al, $72 \%$ percent of children that bruxed showed significant anxiety scores, while only $12 \%$ of nonbruxers had similar psychological findings [9]. This would indicate that the act of bruxing is highly correlated with anxiety disorders. The results from this study agree with

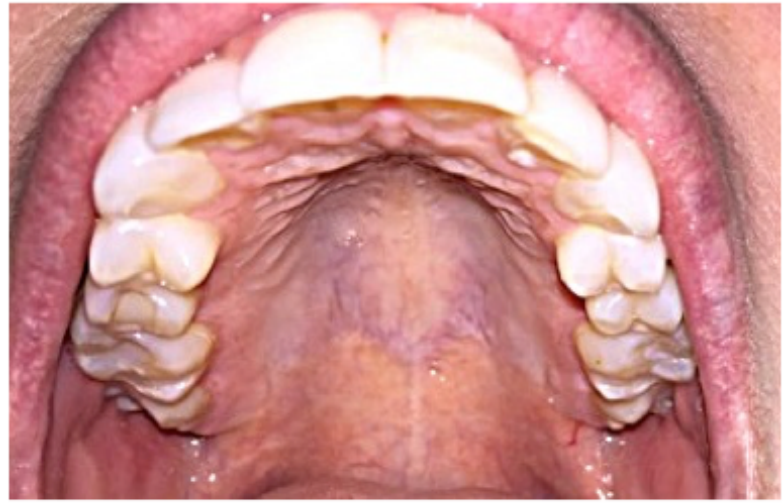

Figure 6: Intraoral signs of bruxism in individual III.3.

the findings from the family reported here. Indeed, in this family, bruxism and multifactorially inherited anxiety disorders appear to be intertwined. Each person with a diagnosed anxiety-related disorder also had signs of occlusal wear associated with bruxism and were aware that they bruxed while awake. Similarly, three out of four of the indi- viduals (I.1, III.4, and III.6) who did not have an anxiety disorder also did not brux. Only one family member

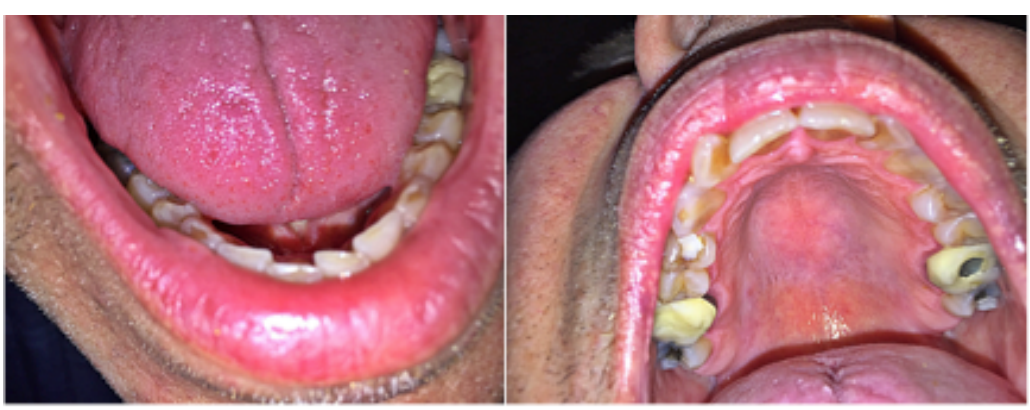

Figure 7. Intraoral signs of bruxism in individual II.6. (individual II.1) showed signs of occlusal trauma from bruxism, but was not diagnosed with anxiety. This family member attributed the wear patterns to "occasional grinding" due to stress in the workplace.

This report highlights the importance for dental health professionals to understand the significance of dental manifestations due to other conditions. Many dental professionals are aware of the evidence linking periodontal disease to heart disease [10] and diabetes [11]. As a result, many dentists are able to act as "early warning indicators" and counsel their patients about possible systemic comorbidities. Similarly, the dental field should become aware of bruxism as a possible predictor of heritable anxiety-related disorders. Anxiety disorders are highly related to suicidal behaviors, particularly in children and adolescents [12]. If dentists are able to recognize the oral mani- festations of anxiety and stress in their patients, they may be able to counsel these patients and ultimately direct them to life-saving services such as therapists and crisis centers.

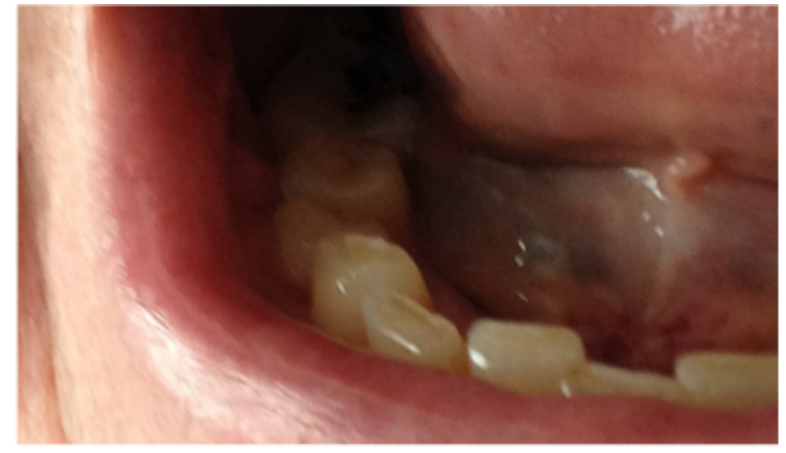

Figure 8. Intraoral signs of bruxism in individual II.1. This individual was the only member of the extended family to have signs of bruxism without a professional diagnosis of an anxiety-related disorder. 


\section{References}

1. Bruxism defined and graded: an international consensus. Lobbezoo F, Ahlberg J, Glaros AG, Kato T, Koyano K, Lavigne GJ, de Leeuw R, Manfredini D, Svensson P, Winocur E. J Oral Rehabil. 2013 Jan;40(1):2-4. doi: 10.1111/joor.12011. Epub 2012 Nov 4. PMID: 23121262

2. Bruxism physiology and pathology: an overview for clinicians. Lavigne GJ, Khoury S, Abe S, Yamaguchi T, Raphael K. J Oral Rehabil. 2008 Jul;35(7):476-94. doi: $10.1111 / \mathrm{j} .1365-$ 2842.2008.01881.x. Review. PMID: 18557915

3. Role of psychosocial factors in the etiology of bruxism. Manfredini D, Lobbezoo F. J Orofac Pain. 2009 Spring;23(2):153-66. Review. PMID: 19492540

4. Genetics of pediatric anxiety disorders. Sakolsky DJ, McCracken JT, Nurmi EL. Child Adolesc Psychiatr Clin N Am. 2012 Jul;21(3):479500. doi: 10.1016/j.chc.2012.05.010. Review. PMID: 22800990

5. A review and meta-analysis of the genetic epidemiology of anxiety disorders. Hettema JM, Neale MC, Kendler KS. Am J Psychiatry. 2001 Oct;158(10):1568-78. PMID: 11578982

6. First genome-wide association study on anxietyrelated behaviours in childhood. Trzaskowski M, Eley TC, Davis OS, Doherty SJ, Hanscombe KB, Meaburn EL, Haworth CM, Price T, Plomin R. PLoS One. 2013;8(4):e58676. doi: 10.1371/journal.pone.0058
676. Epub 2013 Apr 2. PMID: 23565138

Epub 2011 Jul 28. Review. PMID: 21851804
7. Symptoms of anxiety and depression in a volunteer twin population. The etiologic role of genetic and environmental factors.

Kendler KS, Heath A, Martin NG, Eaves LJ. Arch Gen Psychiatry. 1986 Mar;43(3):213-21. PMID: 3954540

8. Bruxism: a literature review. Reddy SV, Kumar MP, Sravanthi D, Mohsin AH, Anuhya V. J Int Oral Health. 2014 Nov-Dec;6(6):105-9. Review. PMID: 25628497

9. The anxiety in bruxer child. A case-control study. Monaco A, Ciammella NM, Marci MC, Pirro R, Giannoni M. Minerva Stomatol. 2002 Jun;51(6):247-50. PMID: 12147977

10. Periodontal disease and coronary heart disease incidence: a systematic review and meta-analysis. Humphrey LL, Fu R, Buckley DI, Freeman M, Helfand M. J Gen Intern Med. 2008 Dec;23(12):2079-86. doi: 10.1007/s11606-008-07876. Epub 2008 Sep 20. Review. PMID: 18807098

11. Diabetes mellitus: biochemical, histological and microbiological aspects in periodontal disease. Marigo L, Cerreto R, Giuliani M, Somma F, Lajolo C, Cordaro M. Eur Rev Med Pharmacol Sci. 2011 Jul;15(7):751-8. Review. PMID: 21780542

12. Suicide-related behaviors and anxiety in children and adolescents: a review. Hill RM, Castellanos D, Pettit JW. Clin Psychol Rev. 2011 Nov;31(7):1133-44. doi: 10.1016/j.cpr.2011.07.008. 